\title{
Surface Multiplasmonics
}

\author{
A. Lakhtakia \\ Pennsylvania State University, USA
}

The planar interface of a metal and a dielectric material can guide multiple surface-plasmon-polariton (SPP) waves - all of the same frequency, but different phase speed, attenuation rate, and spatial profiles of fieldsprovided that the dielectric material is periodically nonhomogeneous in the direction normal to the interface. Theoretical and experimental research during the last four years have validated this concept. Emerging applications of surface multiplasmonics include solar-energy harvesting as well as optical sensing of biological and chemical sensing.

NOTES

\section{General Session II}

\section{Carbon Nanotubes and Graphene as Terahertz Emitters and Detectors}

\author{
M. Portnoi, R. Hartmann, O. Kibis, M. Rosenau da Costa \\ University of Exeter, United Kingdom
}

We propose and justify several schemes utilizing the unique electronic properties of different types of carbon nanotubes for a broad range of applications to terahertz ( $\mathrm{THz}$ ) optoelectronics, including $\mathrm{THz}$ generation by hot electrons in quasi-metallic nanotubes, frequency multiplication in chiral nanotubes, and tuneable $\mathrm{THz}$ radiation detection and optically-pumped $\mathrm{THz}$ emission in armchair nanotubes in a strong magnetic field. Another direction of our research is $\mathrm{THz}$ applications of graphene. As a gapless semiconductor with high carrier mobility, graphene represents an ideal material for detecting $\mathrm{THz}$ radiation. We calculate absorption rates in graphene focusing on the effect of momentum alignment (anisotropy of the distribution function) of photoexcited carriers created by linearly-polarized excitation. In conjunction with a strong angular dependence of the tunnelling probability for graphene $p-n$ junctions, optical alignment of momenta raises the possibility of using graphene $p-n$ junction structures in polarization-sensitive detectors.

NOTES

\section{From nanophotonic devices to nanophotonic integrated circuits: advances and challenges in development of scalable simulation approaches}

\author{
S. Mingaleev ${ }^{1}$, E. Sokolov ${ }^{1}$, A. Simonov ${ }^{1}$, C. Arellano ${ }^{2}$, I. Koltchanov ${ }^{2}$, A. Richter ${ }^{2}$ \\ ${ }^{1}$ VPI Development Center, Belarus; \\ ${ }^{2}$ VPIsystems, Germany
}

Recent advances in nanophotonics allow integration of hundreds (and even thousands in few years) of photonic devices on a single chip. This rapid progress requires development of appropriate simulation tools that would facilitate design and verification of large-scale photonic integrated circuits. Importantly, the simulation techniques developed for modeling nanophotonic devices are badly scalable and become inefficient when applied to modeling large-scale circuits. The latter task requires development of new systemlevel simulation approaches. We show that fully passive photonic circuits can be efficiently modeled in the frequency domain using the S-matrix assembly technique. However, modeling of large-scale *active* photonic circuits presents a real challenge since the commonly used time-domain approach is not scalable. To address this problem, we have developed a new hybrid time-and-frequency-domain modeling (TFDM) approach. Here, we show scalability of this new approach, present our advances in its efficient implementation in the framework of the simulation tool VPIcomponentMaker Photonic Circuits, and discuss the remaining challenges. 